\title{
Condromatose sinovial extensa envolvendo todos os tendões do túnel do tarso: relato de caso*
}

\section{Extensive Synovial Chondromatosis Involving All Flexor Tendons in the Tarsal Tunnel: A Case Report}

\author{
Zachariah Pinter $^{1}$ Ashish Shah ${ }^{1}$ Cesar de Cesar Netto ${ }^{1}$ Walter Smith ${ }^{1}$ Andres O'Daly ${ }^{1}$ \\ Alexandre Leme Godoy-Santos ${ }^{2}$ \\ ${ }^{1}$ Divisão de Cirurgia Ortopédica, Departamento de Cirurgia, \\ University of Alabama, Birmingham, Estado Unidos \\ 2 Faculdade de Medicina, Departamento de Ortopedia, Universidade \\ de São Paulo, São Paulo, SP, Brasil

\begin{abstract}
Address for correspondence Alexandre Leme Godoy-Santos, Faculdade de Medicina, Departamento de Ortopedia, Universidade de São Paulo,
\end{abstract} \\ São Paulo, SP, Brasil (e-mail: alexandrelemegodoy@gmail.com).
}

Rev Bras Ortop 2019;54:78-82.

\section{Resumo \\ Palavras-chave \\ - crondomatose sinovial/diagnóstico por imagem \\ - crondomatose sinovial/cirurgia \\ - crondomatose sinovial/diagnóstico \\ - crondomatose sinovial/patologia \\ - sinovite}

\section{Abstract}

A condromatose sinovial é uma condição rara caracterizada por metaplasia condrogênica benigna do tecido sinovial extra-articular, que geralmente afeta uma única bainha tendínea da mão ou do pé. O objetivo é relatar o caso raro de um paciente com diagnóstico de condromatose sinovial extensa afetando todos os tendões flexores na topografia do túnel do tarso, suas características radiológicas, tratamento cirúrgico e desfecho clínico. Os autores apresentam um caso único de condromatose sinovial extensa em um homem de 48 anos envolvendo as bainhas dos tendões flexor longo do hálux, flexor longo dos dedos e tibial posterior, na topografia do túnel do tarso, com extensão para a região plantar do retropé. O sintoma inicial foi de compressão neurovascular (síndrome do túnel do tarso). A suspeita diagnóstica foi condromatose sinovial com base em evidências radiográficas e de ressonância magnética. O paciente foi submetido a ressecção cirúrgica do tumor, bem como a liberação do túnel tarsal e alongamento do tendão dos gastrocnêmios. O diagnóstico foi confirmado por exame histopatológico do espécime colhido no período intraoperatório. A evolução pósoperatória do paciente ocorreu sem complicações e os sintomas da síndrome do túnel do tarso cessaram. Os autores relatam um caso de condromatose sinovial extensa envolvendo todos os tendões flexores do túnel do tarso, corretamente diagnosticado por achados clínicos e radiológicos e que necessitou de ressecção cirúrgica precoce para evitar complicações neurovasculares em longo prazo.

Synovial chondromatosis is a rare condition characterized by benign chondrogenic meta- plasia of extra-articular synovial tissue. It usually affects a single tendon sheath of

\footnotetext{
Estudo conduzido no Divisão de Cirurgia Ortopédica, Departamento de Cirurgia, University of Alabama at Birmingham (UAB), Birmingham, Estado Unidos.

(D) Alexandre Leme Godoy-Santos's ORCID is https://orcid.org/00000002-6672-1869.
}

\section{received}

July 1,2017

accepted

July 13,2017
DOI https://doi.org/

10.1016/j.rboe.2017.07.012. ISSN $0102-3616$.
Copyright $\odot 2019$ by Sociedade Brasileira License terms de Ortopedia e Traumatologia. Published by Thieme Revnter Publicações Ltda, Rio de Janeiro, Brazil 


\author{
Keywords \\ - synovial \\ chondromatosis/ \\ diagnostic imaging \\ - synovial \\ chondromatosis/ \\ surgery \\ - synovial \\ chondromatosis/ \\ diagnosis \\ - synovial \\ chondromatosis/ \\ pathology \\ - synovitis
}

the hand or foot. This report describes a rare case with extensive synovial chondromatosis affecting all flexor tendons in the tarsal tunnel, its radiological features, surgical treatment, and clinical outcome. The authors present a unique case of extensive synovial chondromatosis in a 48-year-old male involving the tendon sheaths of the flexor hallucis longus, flexor digitorum longus, and posterior tibialis tendons, at the level of the tarsal tunnel, with extension into the plantar aspect of the foot. The patient initially presented with symptoms of tarsal tunnel compression and was found to have a $4-\mathrm{cm}$ mass in the posteromedial aspect of the ankle. The presumptive diagnosis of synovial chondromatosis was made based on radiographic and magnetic resonance imaging evidence. The patient underwent surgical resection of the tumor, as well as tarsal tunnel release and gastrocnemius recession. The diagnosis was confirmed postoperatively by surgical histopathology. The postoperative course of the patient was uncomplicated and his tarsal tunnel symptoms resolved. This represents a case of extensive synovial chondromatosis involving all flexor tendons in the tarsal tunnel that was correctly diagnosed by clinical and imaging findings, which required early surgical resection to avoid long-term neurovascular complications.

\section{Introdução}

A condromatose sinovial é uma doença rara caracterizada por metaplasia condrogênica benigna do tecido sinovial extraarticular. $^{1}$ A transformação da sinóvia leva à formação de corpos cartilaginosos que podem residir na sinóvia em si ou flutuar livremente na bainha tendínea. A doença é relatada com maior frequência em indivíduos entre a terceira e a quinta décadas de vida, com ligeira predileção pelo sexo masculino. ${ }^{2}$

A condromatose sinovial normalmente é uma doença localizada que afeta uma única bainha tendínea da mão ou do pé. A invasão de múltiplas estruturas adjacentes já foi relatada, mas é muito rara. ${ }^{3}$ De modo geral, a presença de uma massa palpável é a queixa inicial, já que, a princípio, a doença normalmente não causa dor ou restrição da amplitude de movimento. Os corpos cartilaginosos tendem a se ossificar, o que gera a aparência radiográfica óbvia. Nos casos sem ossificação, a ressonância magnética (RM) ou a tomografia computadorizada (TC) podem ajudar o diagnóstico. ${ }^{2}$

Apresentamos um caso de condromatose sinovial extensa do flexor longo do hálux (FLH), do flexor longo dos dedos (FLD), do tibial posterior (TP) e da região plantar do retropé em um homem de 48 anos de idade.

\section{Objetivos}

Relatar um caso raro de um paciente com condromatose extensa e acometimento de todos os tendões flexores no túnel do tarso, suas características radiológicas, tratamento cirúrgico e desfecho clínico.

\section{Relato de caso}

Um homem de 48 anos de idade apresentou 6 a 8 semanas de dor no calcanhar plantar do pé direito. Ao primeiro exame, a pele do pé e do tornozelo direitos estava intacta, com mínimo aumento de volume, e uma massa posteromedial de $4 \mathrm{~cm}$ com bordas irregulares foi observada no tornozelo (-Fig. $\mathbf{1}$ ).
O paciente relatou que esta massa crescia lentamente pelos últimos 6 anos. 0 exame mais detalhado revelou diminuição da sensibilidade no calcanhar medial e no aspecto plantar do pé, aumento da sensibilidade plantar na inserção da fáscia plantar e sinal de Tinel positivo no túnel do tarso. A amplitude de movimento do tornozelo e da região subtalar estava dentro dos limites normais. As radiografias do pé e do tornozelo direitos mostraram osteófitos plantares e uma lesão de Haglund, mas sem calcificação evidente da massa (-Fig. 2). A RM ponderada em T2 mostrou uma massa irregular de $4 \mathrm{~cm}$ comprimido o feixe neurovascular. Havia acometimento extenso das bainhas tendíneas do FLD, do FLH e do TP no tornozelo posteromedial, com extensão pela região plantar do retropé (-Fig. 3). No momento do diagnóstico, o paciente optou pelo tratamento conservativo com

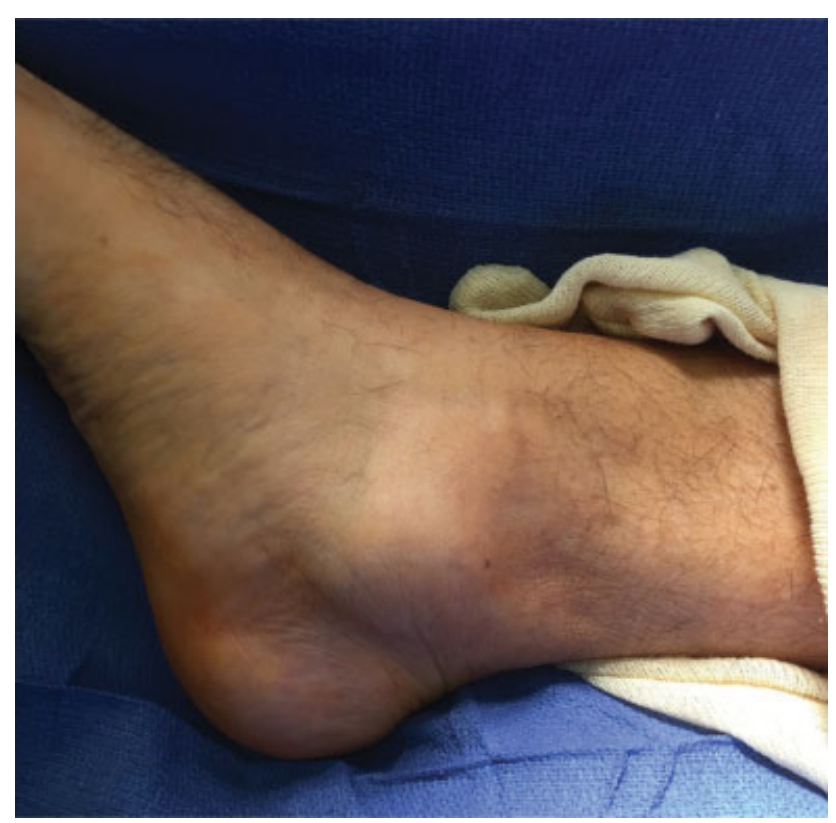

Fig. 1 Grande massa irregular visível sobre o maléolo medial posterior. 

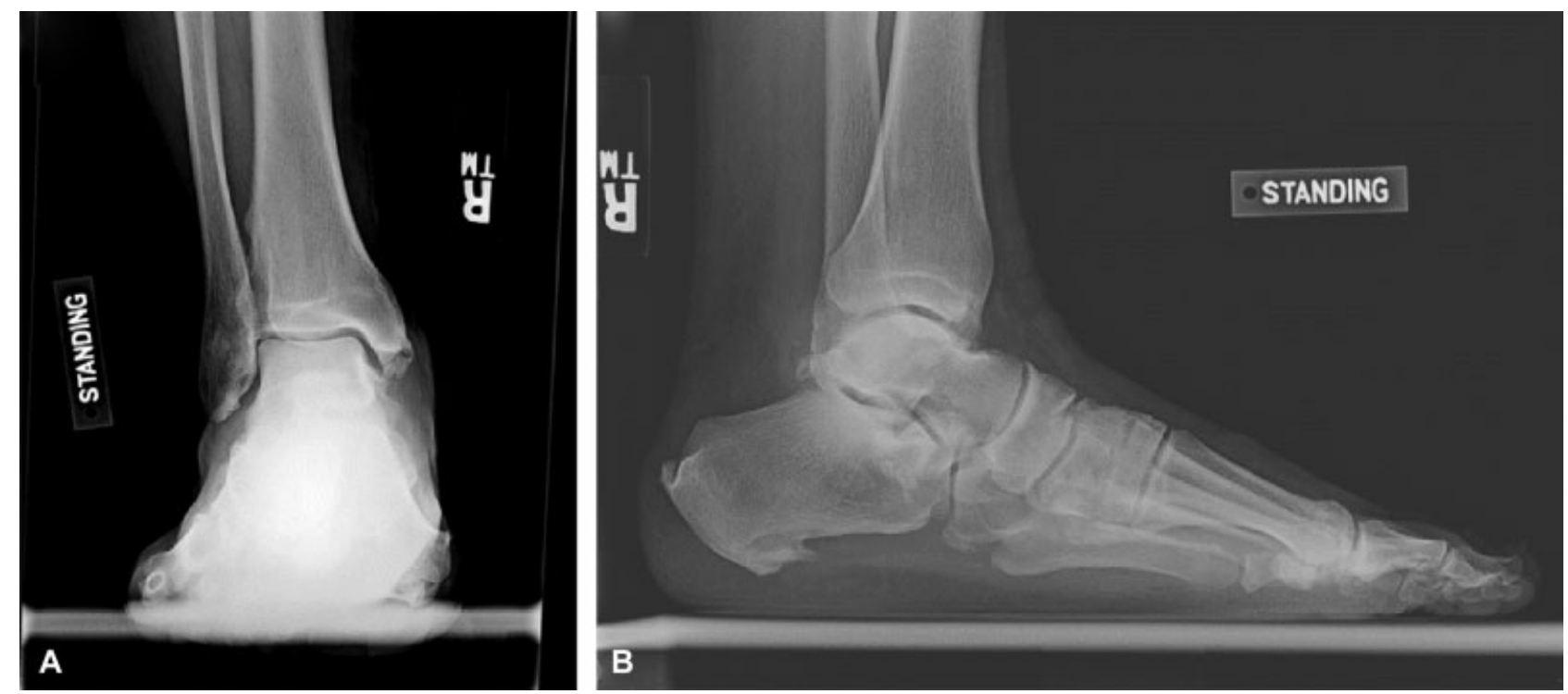

Fig. 2 (A) Radiografia anteroposterior e (B) lateral do tornozelo direito do paciente em pé mostrando a ausência de evidências de calcificação na massa.

bota, anti-inflamatórios não esteroidais e fisioterapia. No entanto, o paciente retornou para o acompanhamento 10 semanas depois se queixando dos mesmos sintomas.

Após exaurir as opções terapêuticas conservadoras, o paciente foi submetido à excisão cirúrgica do tumor. Durante o procedimento, descobriu-se que a massa envolvia a bainha tendínea e cobria os tendões do FLH, do FLD e doTP ( - Fig. 4). A liberação do túnel tarsal e o alongamento do tendão dos gastrocnêmios também foram realizados. A avaliação histopatológica da massa mostrou aglomerados de condrócitos clonais dispostos em lóbulos, com atipia variável e binucleação ocasional, confirmando o diagnóstico de condromatose sinovial (-Fig. 5). O paciente não apresentou complicações no período pós-operatório. Em sua consulta 8 semanas após o procedimento, a dor e os sintomas de túnel do tarso do paciente haviam se resolvido por completo.

Informamos ao paciente que os dados relativos a este caso seriam enviados para publicação e obtivemos seu consenti- mento (aprovado pelo Conselho de Revisão Institucional [CRI] em 29/6/2016 sob o número X160503004).

\section{Discussão}

A condromatose sinovial é uma doença rara, ${ }^{1}$ geralmente observada como uma lesão isolada em uma única bainha tendínea da mão ou do pé.É extremamente raro que a doença apresente acometimento multifocal e se localize na região do tornozelo. Em $90 \%$ das vezes, as radiografias mostram uma massa de tecido mole e calcificações, enquanto a TC revela a presença de calcificação em $100 \%$ dos casos de condromatose tenossinovial. ${ }^{2}$ A RM também auxilia muito o diagnóstico, já que pode revelar características intrínsecas do tecido condroide e trazer novas informações sobre as localizações precisas das lesões.

O diagnóstico diferencial da condromatose sinovial depende do uso das modalidades de imagem anteriormente
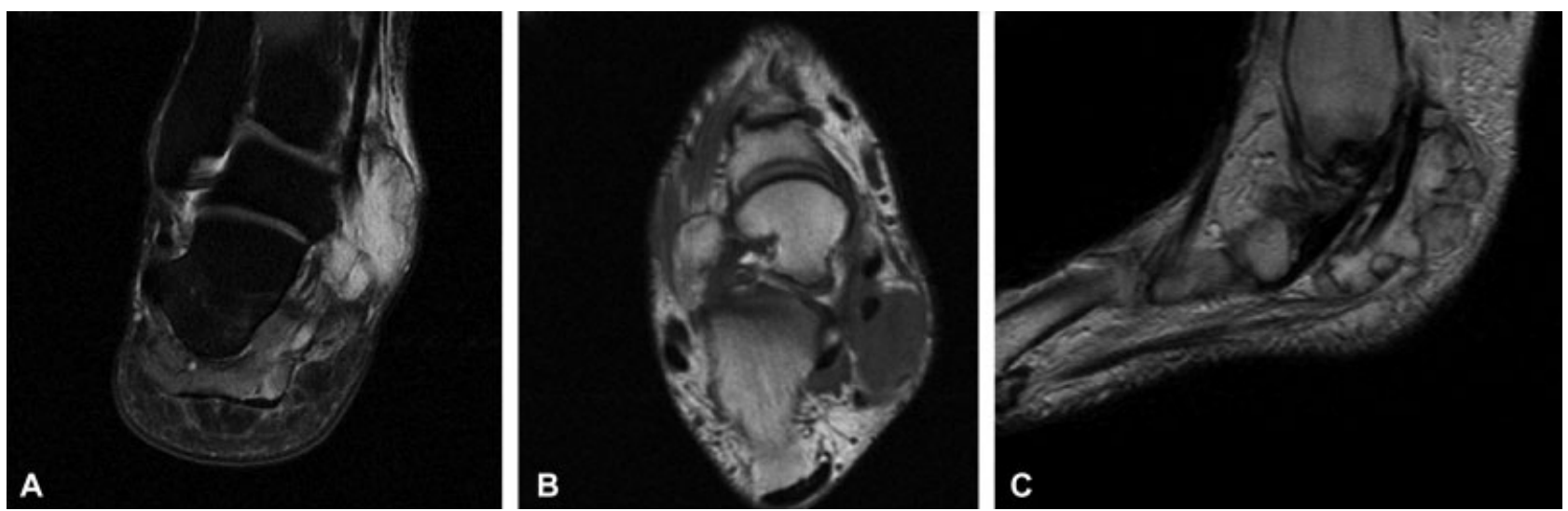

Fig. 3 (A) A imagem coronal de RM ponderada em T2 do tornozelo direito mostra o sinal de alta intensidade que representa a massa posteromedial; (B) a imagem axial da RM ponderada em $\mathrm{T} 1$ do tornozelo direito mostra o sinal de baixa intensidade da massa com acometimento do tendão flexor longo do hálux, do tendão flexor longo dos dedos e do tendão posterior tibial; (C) a imagem sagital da RM ponderada em T1 do tornozelo direito mostra o acometimento posteromedial com extensão pela região plantar do retropé. 

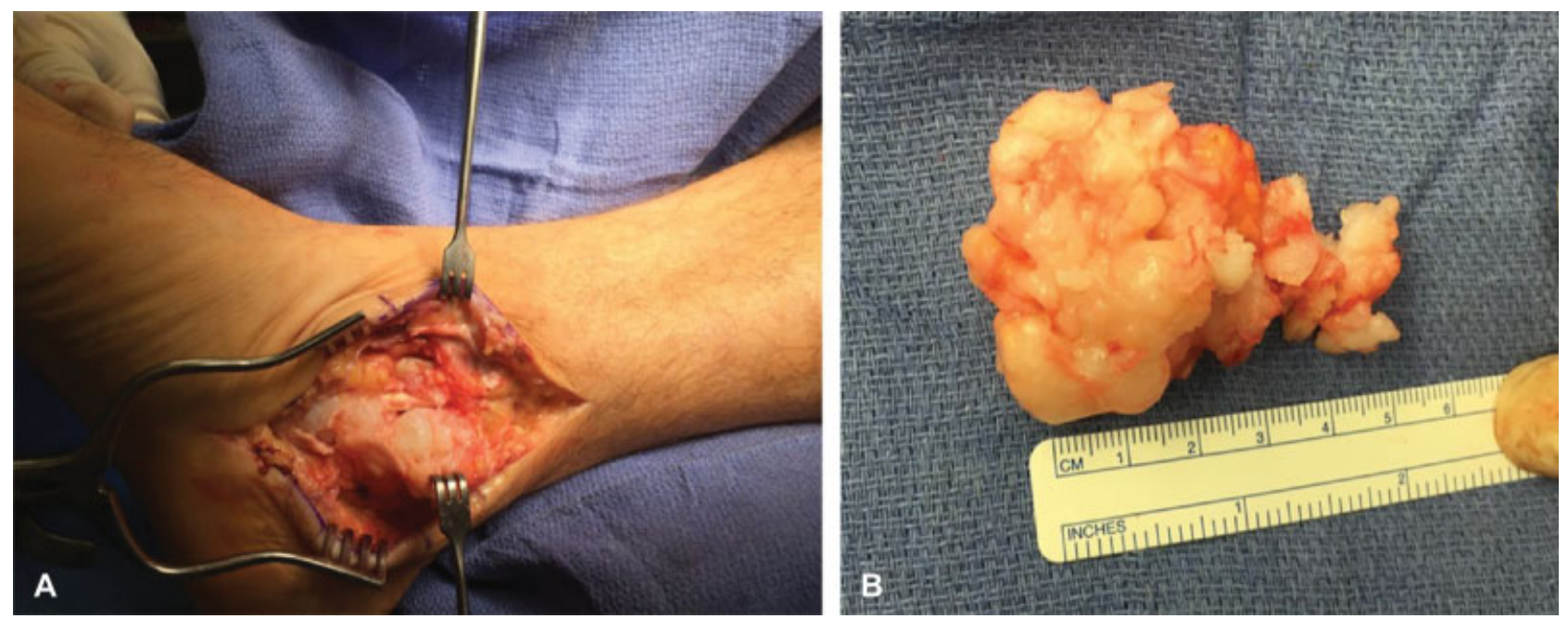

Fig. 4 (A) A imagem intraoperatória mostra o extenso acometimento do tendão pela massa; (B) amostra patológica fresca.

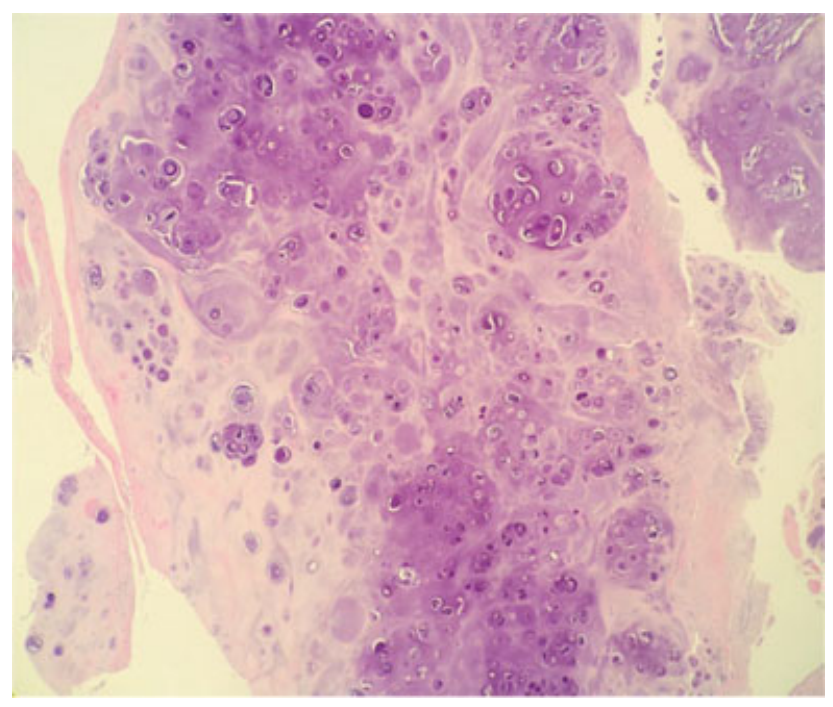

Fig. 5 Cortes histológicos transversais da amostra de condromatose sinovial mostrando os aglomerados de condrócitos e graus variáveis de atipia.

mencionadas na avaliação da doença. A radiografia que revela corpos soltos ossificados e proliferação osteocondromatosa parosteal bizarra deve levar à suspeita de tumor de células gigantes do tornozelo, condrossarcoma ou sarcoma sinovial. Por outro lado, se as radiografias não mostrarem corpos soltos ossificados, mas sim distensão do tecido mole da bainha tendínea, as considerações diferenciais devem incluir tenossinovite, condromatose tenossinovial e tumor de células gigantes tenossinoviais. A RM permite o contraste do detalhe de tecido mole e auxilia a localização precisa das lesões em relação à bainha tendínea. A identificação de bainha tendínea distendida por fluido e preenchida por inúmeros corpos soltos pequenos à RM deve levar à consideração de condromatose sinovial, artrite idiopática juvenil (AIJ) ou artrite reumatoide (AR), que comumente se apresenta com corpos riziformes. A administração intravenosa de gadolínio pode ajudar a diferenciação das alterações infla- matórias, observadas na AIJ ou em infecções. No nosso caso, a RM mostrou uma massa irregular com acometimento da bainha tendínea do FLH, do FLD e do TP e extensão pela região plantar do retropé. Considerando os resultados radiográficos e da RM, o diagnóstico deste caso foi determinado como condromatose tenossinovial.

Até onde tempos conhecimento, houve apenas quarto casos previamente relatados de condromatose sinovial com acometimento da bainha tendínea do $\mathrm{FLH}^{4-7}$ e apenas um destes casos envolveu a tríade de FLH, FLD e túnel do tarso. ${ }^{4}$ No caso com acometimento de FLH, FLD e túnel do tarso, um homem de 23 anos de idade apresentava dor ocasional no local por 13 anos. ${ }^{4}$ Seu diagnóstico inicial foi estabelecido com base nos múltiplos corpos ossificados observados às radiografias.

Em nosso caso, o extenso acometimento de FLH, FLD, TP e túnel do tarso combinado ao grande tamanho do tumor demonstra que o crescimento intenso pode ocorrer antes que o paciente apresente sintomas. Mesmo quando o tumor atinge um tamanho crítico, os sintomas associados geralmente são menores. O aumento de sensibilidade não é incomum, mas a restrição de movimento do tornozelo, normalmente, não é observada. Assim, é essencial que os médicos conheçam a apresentação relativamente indolente desta doença e a ampla variedade de achados de imagem que podem estar presentes. A ressecção cirúrgica é o pilar da terapia; no entanto, a condromatose tenossinovial tem alta taxa de recidiva. Portanto, o acompanhamento cuidadoso é essencial após o tratamento cirúrgico.

Relatamos um caso de condromatose sinovial extensa com acometimento de todos os tendões flexores no túnel tarsal que foi corretamente diagnosticado de acordo com os achados clínicos e de imagem e com necessidade de ressecção cirúrgica rápida para evitar o desenvolvimento de complicações neurovasculares em longo prazo.

\section{Conflitos de interesse}

Os autores declaram não haver conflitos de interesse. 


\section{Referências}

1 Lasmar NP, Vieira RB, Rosa JdeO, Lasmar RCP, Scarpa AC. SYNOVIAL CHONDROMATOSIS. Rev Bras Ortop 2015;45(5): 490-2

2 Walker EA, Murphey MD, Fetsch JF. Imaging characteristics of tenosynovial and bursal chondromatosis. Skeletal Radiol 2011;40 (3):317-25

3 Fetsch JF, Vinh TN, Remotti F, Walker EA, Murphey MD, Sweet DE. Tenosynovial (extraarticular) chondromatosis: an analysis of 37 cases of an underrecognized clinicopathologic entity with a strong predilection for the hands and feet and a high local recurrence rate. Am J Surg Pathol 2003;27(9):1260-8
4 Sugimoto K, Iwai M, Kawate K, Yajima H, Takakura Y. Tenosynovial osteochondromatosis of the tarsal tunnel. Skeletal Radiol 2003;32 (2):99-102

5 Lui TH. Tenosynovial osteochondromatosis of the flexor hallucis longus tendon treated by tendoscopy. J Foot Ankle Surg 2015;54 (4):758-64

6 Thomsen TW, Hogrefe CP, Hall MM, Amendola A. Tenosynovial osteochondromatosis of the flexor hallucis longus in a division I tennis player. Clin J Sport Med 2015;25(6):e74-6

7 Winters NI, Thomson AB, Flores RR, Jordanov MI. Tenosynovial chondromatosis of the flexor hallucis longus in a 17-year-old girl. Pediatr Radiol 2015;45(12):1874-7 